\title{
Structured Low Rank Approximation of a Bezout Matrix
}

\author{
Dongxia Sun and Lihong Zhi \\ Key Laboratory of Mathematics Mechanization \\ Institute of Systems Science, AMSS, Academia Sinica \\ Beijing, 100080, China
}

\begin{abstract}
The task of determining the approximate greatest common divisor (GCD) of more than two univariate polynomials with inexact coefficients can be formulated as computing for a given Bezout matrix a new Bezout matrix of lower rank whose entries are near the corresponding entries of that input matrix. We present an algorithm based on a version of structured nonlinear total least squares (SNTLS) method for computing approximate GCD and demonstrate the practical performance of our algorithm on a diverse set of univariate polynomials.
\end{abstract}

\section{Introduction}

The computation of approximate GCDs of univariate polynomials has been extensively studied in $[21,35,29,7,11,24,18,13,4,33,40,30,34,39,8,37,38,9,10]$. It can be formulated as an optimization problem:

Problem 1.1 Given univariate polynomials $f_{1}, \ldots, f_{l} \in \mathbb{R}[x] \backslash\{0\}$ with $\operatorname{deg}\left(f_{1}\right)=d_{1}, \ldots$, $\operatorname{deg}\left(f_{l}\right)=d_{l}$, assume $d_{1}=\max \left(d_{1}, \ldots, d_{l}\right)$. For a positive integer $k, k \leq \min \left(d_{1}, \ldots, d_{l}\right)$, we wish to compute $\Delta f_{1}, \ldots, \Delta f_{l} \in \mathbb{R}[x]$ such that $\operatorname{deg}\left(\Delta f_{1}\right) \leq d_{1}, \ldots, \operatorname{deg}\left(\Delta f_{l}\right) \leq d_{l}$, $\operatorname{deg}\left(\operatorname{GCD}\left(f_{1}+\Delta f_{1}, \ldots, f_{l}+\Delta f_{l}\right)\right) \geq k$ and $\left\|\Delta f_{1}\right\|_{2}^{2}+\cdots+\left\|\Delta f_{l}\right\|_{2}^{2}$ is minimized.

In $[21,22,23]$, the authors transformed the above problem into computing for a generalized Sylvester matrix the nearest singular matrix with the generalized Sylvester structure. They presented iterative algorithms based on structured total least norm algorithms in [32, 31, 25, $26]$ to solve the optimization problem. It is well known that Bezout matrix can also be used to compute GCDs, see recent paper [10] and references there. Compared with the generalized Sylvester matrix, Bezout matrix has smaller size. However, entries of the Bezout matrix are bilinear in coefficients of the polynomials. Hence, we propose to apply the structured nonlinear total least squares (SNTLS) algorithm [19, 26] to compute the nearest singular matrix with Bezout structure. We show how to solve PROBLEM 1.1, at least for a local minimum, by applying SNTLS with $L_{2}$ norm to a submatrix of the Bezout matrix.

We organize the paper as follows. In Section 2, we introduce some notations and discuss the equivalence between the GCD problem and the low rank approximation of a matrix with Bezout structure. In Section 3, we consider solving an overdetermined system with Bezout structure based on SNTLS. In Section 4, we describe our algorithm for two examples

\footnotetext{
${ }^{1)}$ Partially supported by a National Key Basic Research Project of China 2004CB318000 and Chinese National Science Foundation under Grant 10401035.
} 
and compare our results with previous work in $[21,22,23]$. We conclude in Section 5 with remarks on the complexity and the rate of convergence of our algorithm.

\section{2. preliminaries}

The GCD problems are closely related with structured matrices, such as Sylvester matrix, Bezout matrix, Hankel matrix and so forth. In the following subsections we introduce the structured matrices.

\subsection{Bezout Matrix}

Suppose we are given two univariate polynomials $f_{1}, f_{2} \in \mathbb{R}[x] \backslash\{0\}$ with $\operatorname{deg}\left(f_{1}\right)=m$ and $\operatorname{deg}\left(f_{2}\right)=n$, we assume $m \geq n$,

$$
\begin{array}{ll}
f_{1}=u_{m} x^{m}+u_{m-1} x^{m-1}+\cdots+u_{1} x+u_{0}, & u_{m} \neq 0, \\
f_{2}=v_{n} x^{n}+v_{n-1} x^{n-1}+\cdots+v_{1} x+v_{0}, & v_{n} \neq 0 .
\end{array}
$$

The Bezout matrix $\hat{B}\left(f_{1}, f_{2}\right)=\left(\hat{b}_{i j}\right)$ is defined by

$$
\hat{b}_{i j}=\left|u_{0} v_{i+j-1}\right|+\left|u_{1} v_{i+j-2}\right|+\cdots+\left|u_{k} v_{i+j-k-1}\right|,
$$

where $\left|u_{r} v_{s}\right|=u_{r} v_{s}-u_{s} v_{r}, k=\min (i-1, j-1)$ and $v_{r}=0$ if $r>n[3,17]$. It satisfies that

$$
\frac{f_{1}(x) f_{2}(y)-f_{1}(y) f_{2}(x)}{x-y}=\left[1, x, x^{2}, \ldots, x^{m-1}\right] \hat{B}\left(f_{1}, f_{2}\right)\left[1, y, y^{2}, \ldots, y^{m-1}\right]^{T} .
$$

Notice that the Bezout matrix $B\left(f_{1}, f_{2}\right)$ defined in Maple is as follows:

$$
B\left(f_{1}, f_{2}\right)=-J \hat{B}\left(f_{1}, f_{2}\right) J
$$

where $J$ is an anti-diagonal matrix with 1 as its nonzero entries.

The Bezout matrix can be generalized for nonzero univariate polynomials $f_{1}, \ldots, f_{l} \in$ $\mathbb{R}[x] \backslash\{0\}$ with $\operatorname{deg}\left(f_{1}\right)=d_{1}, \ldots, \operatorname{deg}\left(f_{l}\right)=d_{l}$. Suppose $d_{1}=\max \left(d_{1}, \ldots, d_{l}\right), B\left(f_{1}, \ldots, f_{l}\right) \in$ $\mathbb{R}^{(l-1) d_{1} \times d_{1}}$ is defined by

$$
B\left(f_{1}, \ldots, f_{l}\right)=\left[\begin{array}{c}
B\left(f_{1}, f_{2}\right) \\
B\left(f_{1}, f_{3}\right) \\
\vdots \\
B\left(f_{1}, f_{l}\right)
\end{array}\right]
$$

Suppose $l=2$ and $m=n=4$, the Sylvester matrix $S\left(f_{1}, f_{2}\right)$ and Bezout matrix $B\left(f_{1}, f_{2}\right)$ defined in Maple are:

$$
S\left(f_{1}, f_{2}\right)=\left[\begin{array}{cccccccc}
u_{4} & u_{3} & u_{2} & u_{1} & u_{0} & 0 & 0 & 0 \\
0 & u_{4} & u_{3} & u_{2} & u_{1} & u_{0} & 0 & 0 \\
0 & 0 & u_{4} & u_{3} & u_{2} & u_{1} & u_{0} & 0 \\
0 & 0 & 0 & u_{4} & u_{3} & u_{2} & u_{1} & u_{0} \\
v_{4} & v_{3} & v_{2} & v_{1} & v_{0} & 0 & 0 & 0 \\
0 & v_{4} & v_{3} & v_{2} & v_{1} & v_{0} & 0 & 0 \\
0 & 0 & v_{4} & v_{3} & v_{2} & v_{1} & v_{0} & 0 \\
0 & 0 & 0 & v_{4} & v_{3} & v_{2} & v_{1} & v_{0}
\end{array}\right]
$$




$$
\begin{aligned}
& B\left(f_{1}, f_{2}\right)= \\
& \quad\left[\begin{array}{cccc}
u_{3} v_{4}-u_{4} v_{3} & u_{2} v_{4}-u_{4} v_{2} & u_{1} v_{4}-u_{4} v_{1} & u_{0} v_{4}-u_{4} v_{0} \\
u_{2} v_{4}-u_{4} v_{2} & u_{2} v_{3}-u_{3} v_{2}+u_{1} v_{4}-u_{4} v_{1} & u_{1} v_{3}-u_{3} v_{1}+u_{0} v_{4}-u_{4} v_{0} & u_{0} v_{3}-u_{3} v_{0} \\
u_{1} v_{4}-u_{4} v_{1} & u_{1} v_{3}-u_{3} v_{1}+u_{0} v_{4}-u_{4} v_{0} & u_{1} v_{2}-u_{2} v_{1}+u_{0} v_{3}-u_{3} v_{0} & u_{0} v_{2}-u_{2} v_{0} \\
u_{0} v_{4}-u_{4} v_{0} & u_{0} v_{3}-u_{3} v_{0} & u_{0} v_{2}-u_{2} v_{0} & u_{0} v_{1}-u_{1} v_{0}
\end{array}\right]
\end{aligned}
$$

The size of the Sylvester matrix is larger, while the entries of the Bezout matrix are bilinear in coefficients of $f_{1}$ and $f_{2}$. When the degrees of the polynomials are high, the advantage of the Bezout matrix is obvious.

\subsection{Hankel matrix}

A Hankel matrix $H \in \mathbb{R}^{m \times m}$ is formed from $2 m-1$ real numbers $\left(h_{i}\right)_{i=0}^{2 m-2}$ :

$$
H\left(f_{1}, f_{2}\right)=\left[\begin{array}{cccc}
h_{0} & h_{1} & \ldots & h_{m-1} \\
h_{1} & h_{2} & \ldots & h_{m} \\
\vdots & \vdots & \ddots & \vdots \\
h_{m-1} & h_{m} & \ldots & h_{2 m-2}
\end{array}\right]
$$

Given two univariate polynomials $f_{1}, f_{2} \in \mathbb{R}[x] \backslash\{0\}$ with $\operatorname{deg}\left(f_{1}\right)=m$ and $\operatorname{deg}\left(f_{2}\right)=n$, where $m>n$, assume $R(x)=\frac{f_{2}(x)}{f_{1}(x)}=\sum_{i=0}^{+\infty} h_{i} x^{-i-1}$. The vector $\left(h_{i}\right)_{i=0, \cdots,+\infty}$ forms a Hankel matrix $H_{\infty}$. The m-th leading principal submatrix $H\left(f_{1}, f_{2}\right)$ is defined as the Hankel matrix of $f_{1}$ and $f_{2}$. Given the coefficients of $f_{1}$ and $f_{2}$, the entries of the Hankel matrix $H\left(f_{1}, f_{2}\right)$ can be computed [6].

Proposition 2.1 $[15,14,6]$ Given two univariate polynomials $f_{1}, f_{2} \in \mathbb{R}[x] \backslash\{0\}$ with $\operatorname{deg}\left(f_{1}\right)=m, \operatorname{deg}\left(f_{2}\right)=n(m>n)$, then we have:

$$
B\left(f_{1}, f_{2}\right)=-B\left(f_{1}, 1\right) J H\left(f_{1}, f_{2}\right) J B\left(f_{1}, 1\right)
$$

Proposition 2.2 [6] Given two univariate monic polynomials $f_{1}, f_{2} \in \mathbb{R}[x] \backslash\{0\}$ with $\operatorname{deg}\left(f_{1}\right)=$ $m$ and $\operatorname{deg}\left(f_{2}\right)=n(m>n)$, let $c(x)=\operatorname{GCD}\left(f_{1}(x), f_{2}(x)\right)$ be a monic polynomial of degree $m-k$. Then we have:

- $\operatorname{rank}\left(H\left(f_{1}, f_{2}\right)\right)=k, \operatorname{det}\left(H_{k}\right) \neq 0$, where $H_{i}$ is the $i \times i$ leading principal submatrix of $H\left(f_{1}, f_{2}\right), \operatorname{det}\left(H_{i}\right)=0$ for $i>k$;

- If $H_{k+1} \mathbf{w}=0, \mathbf{w}=\left(w_{i}\right)_{i=0, \cdots, k}$, that is, if

$$
H_{k}\left(\begin{array}{c}
w_{0} \\
\vdots \\
w_{k-1}
\end{array}\right)=-w_{k}\left(\begin{array}{c}
h_{k} \\
\vdots \\
h_{2 k-1}
\end{array}\right)
$$

then

$$
f_{1}(x)=c(x) \sum_{i=0}^{k} w_{i} x^{i}
$$


Remark 2.3 [6] Since $B\left(f_{1}, 1\right) J$ and $J B\left(f_{1}, 1\right)$ are lower triangular and upper triangular matrices respectively, we deduce from the relation $B\left(f_{1}, f_{2}\right)=-B\left(f_{1}, 1\right) J H\left(f_{1}, f_{2}\right) J B\left(f_{1}, 1\right)$, that $B\left(f_{1}, f_{2}\right) \mathbf{y}=0, y_{k}=1, y_{i}=0, i>k$, if and only if $\mathbf{w}=J B\left(f_{1}, 1\right) y$, where $\mathbf{w}$ is the vector of Proposition 2.2. Moreover, $\operatorname{det}\left(B\left(f_{1}, f_{2}\right)\right)_{k} \neq 0$, and $\operatorname{det}\left(B\left(f_{1}, f_{2}\right)\right)_{i}=0$, for $i>k$, where $\left(B\left(f_{1}, f_{2}\right)\right)_{i}$ is the $i \times i$ leading principal submatrix of $B\left(f_{1}, f_{2}\right)$.

Remark 2.4 Notice that the Propositions 2.1,2.2 are also correct for $m=n$. Let $\bar{f}_{2}(x)=$ $f_{2}(x)-\frac{v_{n}}{u_{m}} f_{1}(x)$, and $R(x)=\frac{f_{2}(x)}{f_{1}(x)}=\frac{v_{n}}{u_{m}}+\frac{\bar{f}_{2}(x)}{f_{1}(x)}=h_{0}+\sum_{i=1}^{+\infty} h_{i} x^{-i}$ where $h_{0}=\frac{v_{n}}{u_{m}}$. The matrix $H\left(f_{1}, f_{2}\right)$ is defined as:

$$
H\left(f_{1}, f_{2}\right)=\left[\begin{array}{cccc}
h_{1} & h_{2} & \ldots & h_{m} \\
h_{2} & h_{3} & \ldots & h_{m+1} \\
\vdots & \vdots & \ddots & \vdots \\
h_{m} & h_{m+1} & \ldots & h_{2 m-1}
\end{array}\right]
$$

Hence $B\left(f_{1}, f_{2}\right)=B\left(f_{1}, \bar{f}_{2}\right)$ and $H\left(f_{1}, f_{2}\right)=H\left(f_{1}, \bar{f}_{2}\right)$.

Corollary 2.5 Given univariate polynomials $f_{1}, \ldots, f_{l} \in \mathbb{R}[x] \backslash\{0\}$ with $\operatorname{deg}\left(f_{1}\right)=d_{1}, \cdots$, $\operatorname{deg}\left(f_{l}\right)=d_{l}, d_{1}=\max \left(d_{1}, d_{2}, \cdots, d_{l}\right)$, then we have:

$$
B\left(f_{1}, \cdots, f_{l}\right)=\left[\begin{array}{c}
B\left(f_{1}, f_{2}\right) \\
B\left(f_{1}, f_{3}\right) \\
\vdots \\
B\left(f_{1}, f_{l}\right)
\end{array}\right]_{(l-1) d_{1} \times d_{1}}=-\Gamma H\left(f_{1}, \cdots, f_{l}\right) J B\left(f_{1}, 1\right),
$$

where

$$
\Gamma=\left[\begin{array}{lll}
B\left(f_{1}, 1\right) J & & \\
& \ddots & \\
& & B\left(f_{1}, 1\right) J
\end{array}\right]_{(l-1) d_{1} \times\left((l-1) d_{1}\right.}
$$

and

$$
H\left(f_{1}, \cdots, f_{l}\right)=\left[\begin{array}{c}
H\left(f_{1}, f_{2}\right) \\
H\left(f_{1}, f_{3}\right) \\
\vdots \\
H\left(f_{1}, f_{l}\right)
\end{array}\right]_{(l-1) d_{1} \times d_{1}} .
$$

Proof: The proof is derived from Proposition 2.1.

Lemma 2.6 [12] Let $H_{\infty}$ be an infinity Hankel matrix of rank $r$, then we have:

$$
\operatorname{det}\left(H\left(f_{1}, f_{2}\right)_{r}\right) \neq 0 .
$$

Lemma 2.7 [12] Let $H_{\infty}\left(f_{1}, f_{2}\right)$ be an infinity Hankel matrix which is formed from two polynomials $f_{1}, f_{2}$ with degrees $m, n(m \geq n)$ respectively, then

$$
\operatorname{rank}\left(H_{\infty}\left(f_{1}, f_{2}\right)\right) \leq m .
$$




\subsection{Main Theorems}

The following three theorems summarize the relationship between the greatest common divisor(GCD) of $f_{1}, \ldots, f_{l}$ and the Bezout matrix $B\left(f_{1}, \ldots, f_{l}\right)$.

Theorem 2.8 Given univariate polynomials $f_{1}, \ldots, f_{l} \in \mathbb{R}[x] \backslash\{0\}$ with $\operatorname{deg}\left(f_{1}\right)=d_{1}, \ldots$, $\operatorname{deg}\left(f_{l}\right)=d_{l}, d_{1}=\max \left(d_{1}, \ldots, d_{l}\right)$, then we have $\operatorname{dim}\left(\operatorname{Ker} B\left(f_{1}, \ldots, f_{l}\right)\right)$ being equal to the degree of the $G C D$ of $f_{1}, \ldots, f_{l}$.

Proof: A detailed proof is given in [9] .

Theorem 2.9 Given univariate polynomials $f_{1}, \ldots, f_{l} \in \mathbb{R}[x] \backslash\{0\}$ with $\operatorname{deg}\left(f_{1}\right)=d_{1}, \ldots$, $\operatorname{deg}\left(f_{l}\right)=d_{l}, d_{1}=\max \left(d_{1}, \ldots, d_{l}\right)$, then the degree of the $G C D$ of $f_{1}, \ldots, f_{l}$ is at least $k$ for $k \leq \min \left(d_{1}, \ldots, d_{l}\right)$ if and only if the first $d_{1}-k+1$ columns of $B\left(f_{1}, \ldots, f_{l}\right)$ are linearly dependent.

Proof: $\quad$ For $l=2$, since $B\left(f_{1}, 1\right) J$ and $J B\left(f_{1}, 1\right)$ are lower triangular and upper triangular matrices respectively, by Proposition 2.1, we have $\operatorname{rank}\left(B\left(f_{1}, f_{2}\right)_{i}\right)=\operatorname{rank}\left(H\left(f_{1}, f_{2}\right)_{i}\right)$. If the first $d_{1}-k+1$ columns of $B\left(f_{1}, f_{2}\right)$ are linearly dependent, then the first $d_{1}-k+i(i \geq$ 1) columns of $H\left(f_{1}, f_{2}\right)$ are linearly dependent. By Lemma 2.6 and Lemma 2.7, we have $\operatorname{rank}\left(B\left(f_{1}, f_{2}\right)\right) \leq d_{1}-k$. The proof can be derived by Theorem 2.8 and generalized to the cases $l>2$ by Corollary 2.5 .

Theorem 2.10 Given univariate polynomials $f_{1}, \ldots, f_{l} \in \mathbb{R}[x] \backslash\{0\}$ with $\operatorname{deg}\left(f_{1}\right)=d_{1}, \ldots$, $\operatorname{deg}\left(f_{l}\right)=d_{l}, d_{1}=\max \left(d_{1}, \ldots, d_{l}\right)$, let $c(x)=\operatorname{GCD}\left(f_{1}(x), \ldots, f_{l}(x)\right)$ be a polynomial of degree $k$, then we have $\operatorname{rank}\left(B\left(f_{1}, \ldots, f_{l}\right)\right)=d_{1}-k$. Suppose $\mathbf{y}=\left(y_{0}, y_{1}, \ldots, y_{d_{1}-k-1}\right)^{T}$ satisfies $C \mathbf{y}=\mathbf{b}$, where $C$ consists of the first $d_{1}-k$ columns of $B\left(f_{1}, \ldots, f_{l}\right)$, and $\mathbf{b}$ is a vector formed from the $d_{1}-k+1$-th column of $B\left(f_{1}, \ldots, f_{l}\right)$. Let $\mathbf{w}=\left[w_{0}, \ldots, w_{d_{1}-k}\right]^{T}=$ $\left(J B\left(f_{1}, 1\right)\right)_{d_{1}-k+1}\left[y_{0}, \ldots, y_{d_{1}-k}\right]^{T}$, where $y_{d_{1}-k}=-1,\left(J B\left(f_{1}, 1\right)\right)_{d_{1}-k+1}$ is the leading principal $d_{1}-k+1$-th submatrix, then $f_{1}(x)=c(x) w(x)$, with $w(x)=\sum_{i=0}^{d_{1}-k} w_{i} x^{i}$.

See $[1,2,3,15,6,27,5,16,9,10]$ for detailed proofs.

\section{SNTLS for Overdetermined System with Bezout Structure}

The Bezout matrix $B\left(f_{1}, \ldots, f_{l}\right)$ can be parameterized by a vector $\zeta$ which contains the coefficients of $f_{1}, \ldots, f_{l}$. By applying Theorem 2.8, we can transfer the PROBLEM 1.1 into solving the following minimization problem:

$$
\min _{\Delta \mathbf{s} \in \mathbb{R}^{d+l}}\|\Delta \mathbf{s}\|_{2} \quad \text { with } \quad \operatorname{dim}(\operatorname{Ker} B(\mathbf{s}+\Delta \mathbf{s})) \geq k,
$$

where $d=\sum_{i=1}^{l} d_{i}$ and $\mathbf{s}$ is fixed to the initial coefficient vector. Let $B_{k}(\zeta)=\left[D_{1}(\zeta), \mathbf{b}(\zeta), D_{2}(\zeta)\right]$ be the first $d_{1}-k+1$ columns of the Bezout matrix $B(\zeta)$ and let $A(\zeta)=\left[D_{1}(\zeta), D_{2}(\zeta)\right] \in$ $\mathbb{R}^{d_{1}(l-1) \times\left(d_{1}-k\right)}$. According to Theorem 2.9, the minimization problem (15) can be transferred into the following structured nonlinear total least squares problem:

$$
\min _{\Delta \mathbf{s} \in \mathbb{R}^{d+l}}\|\Delta \mathbf{s}\|_{2} \quad \text { with } \quad A(\mathbf{s}+\Delta \mathbf{s}) \mathbf{x}=\mathbf{b}(\mathbf{s}+\Delta \mathbf{s}), \quad \text { for some vector } \quad \mathbf{x} .
$$


Similar to $[21,22,23]$, we choose that column as $\mathbf{b}(\zeta) \in \mathbb{R}^{d_{1}(l-1) \times 1}$ for which the corresponding component in the first right singular vector of $B_{k}$ was maximum in absolute value. In the following, we illustrate how to find the minimum solution of (16) using the structured nonlinear total least squares (SNTLS) method.

We can initialize $\mathbf{x}$ as the unstructured least squares solution $A(\mathbf{s}) \mathbf{x}=\mathbf{b}(\mathbf{s})$ for the input parameters $\mathbf{s}$ and $\Delta \mathbf{s}=\mathbf{0}$. However, as pointed by [25, Section 4.5.3] and [22, 23], another way is to initialize $\Delta \mathbf{s}$ and $\mathbf{x}$ such that they satisfy the nonlinear constrains approximately, $A(\mathbf{s}+\Delta \mathbf{s}) \mathbf{x} \approx b(\mathbf{s}+\Delta \mathbf{s})$. We compute $\Delta \mathbf{s}$ as follows:

$$
\Delta \mathbf{s}=-Y^{T}(\mathbf{s}, \mathbf{v})\left(Y(\mathbf{s}, \mathbf{v}) Y^{T}(\mathbf{s}, \mathbf{v})\right)^{-1} B_{k}(\mathbf{s}) \mathbf{v},
$$

where $\mathbf{v}$ is the right singular vector corresponding to the smallest singular value of $B_{k}(\mathbf{s})$ and matrix $Y$ is the Jacobian of $B_{k}(\zeta) \mathbf{v}$ with respect to $\zeta$, we have

$$
B_{k}(\mathbf{s}+\Delta \mathbf{s}) \mathbf{v}=B_{k}(\mathbf{s}) \mathbf{v}+Y(\mathbf{s}, \mathbf{v}) \Delta \mathbf{s}+O\left(\|\Delta \mathbf{s}\|_{2}^{2}\right)=O\left(\|\Delta \mathbf{s}\|_{2}^{2}\right) .
$$

Suppose $b(\mathbf{s})$ is the $t$-th column corresponding to the absolutely largest component in $\mathbf{v}$; We initialize the vector $\mathbf{x}$ by normalizing the vector $\mathbf{v}$ to make $\mathbf{v}[t]=-1$, i.e.,

$$
\mathbf{x}=\left[-\frac{\mathbf{v}[1]}{\mathbf{v}[t]}, \ldots,-\frac{\mathbf{v}[t-1]}{\mathbf{v}[t]},-\frac{\mathbf{v}[t+1]}{\mathbf{v}[t]}, \ldots\right]^{T} .
$$

We have $A(\mathbf{s}+\Delta \mathbf{s}) \mathbf{x}-b(\mathbf{s}+\Delta \mathbf{s})=O\left(\|\Delta \mathbf{s}\|_{2}^{2}\right)$. Since the initial values of $\Delta \mathbf{s}$ and $\mathbf{x}$ only satisfy the first order of the nonlinear constrains, for the second initialization method to be successful, we usually require that the initial perturbation (17) $\|\Delta \mathbf{s}\|_{2} \ll 1$.

By introducing the Lagrangian multipliers, and neglecting the second-order terms in $\Delta \mathbf{s}$, the constrained minimization problem can be transformed into an unconstrained optimization problem $[26,19]$,

$$
L(\Delta \mathbf{s}, \mathbf{x}, \lambda)=\frac{1}{2} \Delta \mathbf{s}^{T} \Delta \mathbf{s}-\lambda^{T}(\mathbf{b}-A \mathbf{x}-X \Delta \mathbf{s}),
$$

where $X(\zeta, \mathbf{x})$ is the Jacobian of $\mathbf{r}(\zeta, \mathbf{x})=A(\zeta) \mathbf{x}-\mathbf{b}$ with respect to $\zeta$ :

$$
X(\zeta, \mathbf{x})=\nabla_{\zeta}(A(\zeta) \mathbf{x})-\nabla_{\zeta}(\mathbf{b}(\zeta))=\sum_{j=1}^{d_{1}-k} x_{j} \nabla_{\zeta} a_{j}(\zeta)-\nabla_{\zeta}(\mathbf{b}(\zeta))
$$

where $a_{j}(\zeta)$ represents the $j$-th column of $A(\zeta)$. Applying the Newton method on the Lagrangian $L$ yields:

$$
\begin{aligned}
{\left[\begin{array}{ccc}
I_{d+l} & \mathbf{0}_{(d+l) \times\left(d_{1}-k\right)} & X(\mathbf{s}+\Delta \mathbf{s}, \mathbf{x})^{T} \\
\mathbf{0}_{\left(d_{1}-k\right) \times(d+l)} & \mathbf{0}_{\left(d_{1}-k\right) \times\left(d_{1}-k\right)} & A(\mathbf{s}+\Delta \mathbf{s})^{T} \\
X(\mathbf{s}+\Delta \mathbf{s}, \mathbf{x}) & A(\mathbf{s}+\Delta \mathbf{s}) & \mathbf{0}_{(l-1) d_{1} \times(l-1) d_{1}}
\end{array}\right]\left[\begin{array}{c}
\Delta \tilde{\mathbf{s}} \\
\Delta \tilde{\mathbf{x}} \\
\Delta \tilde{\lambda}
\end{array}\right]=} \\
-\left[\begin{array}{c}
\Delta \mathbf{s}+X(\mathbf{s}+\Delta \mathbf{s}, \mathbf{x})^{T} \lambda \\
A(\mathbf{s}+\Delta \mathbf{s})^{T} \lambda \\
A(\mathbf{s}+\Delta \mathbf{s}) \mathbf{x}-\mathbf{b}(\mathbf{s}+\Delta \mathbf{s})
\end{array}\right],
\end{aligned}
$$


where $d=\sum_{i=1}^{l} d_{i}$. The iterative update $\mathbf{x}=\mathbf{x}+\Delta \tilde{\mathbf{x}}, \lambda=\lambda+\Delta \tilde{\lambda}, \mathbf{s}=\mathbf{s}+\Delta \mathbf{s}+\Delta \tilde{\mathbf{s}}$ is stopped when $\|\Delta \tilde{\mathbf{x}}\|_{2}$ and/or $\|\Delta \tilde{\mathbf{s}}\|_{2}$ and/or $\|\Delta \tilde{\lambda}\|_{2}$ becomes smaller than a given tolerance.

Suppose $\tilde{B}_{k}$ is the nearest singular matrix with Bezout structure computed successfully by SNTLS algorithm. Let the perturbed polynomials be $\tilde{f}_{1}, \ldots, \tilde{f}_{l}$. Suppose $k=$ $\operatorname{deg}\left(\operatorname{GCD}\left(\tilde{f}_{1}, \ldots, \tilde{f}_{l}\right)\right)$, the polynomial $\left.c(x)=\operatorname{GCD}\left(\tilde{f}_{1}, \ldots, \tilde{f}_{l}\right)\right)$ can be computed according to Theorem 2.10. However, we can also use the vector $\mathbf{x}$ returned from the iterations (21) to compute the GCD directly. Let $\mathbf{y}=\left[x_{1}, \ldots, x_{t-1},-1, x_{t}, \ldots, x_{d_{1}-k}\right]^{T}$, compute the vector $\mathbf{w}=\left[w_{0}, \ldots, w_{d_{1}-k}\right]^{T}=\left(J B\left(\tilde{f}_{1}, 1\right)\right)_{d_{1}-k+1} \mathbf{y}^{T}$, where $\left(J B\left(\tilde{f}_{1}, 1\right)\right)_{d_{1}-k+1}$ is the leading principal $d_{1}-k+1$-th submatrix. The polynomial $c(x)$ is computed by dividing the polynomial $\tilde{f}_{1}(x)$ by the polynomial $w(x)=\sum_{i=0}^{d_{1}-k} w_{i} x^{i}$, and is returned as the approximate GCD of $f_{1}, \ldots, f_{l}$.

Remark 3.1 When $k$ is smaller than $\operatorname{deg}\left(\operatorname{GCD}\left(\tilde{f}_{1}, \ldots, \tilde{f}_{l}\right)\right)$, as suggested in [23], we may increase $k$ by $k+1$ and run our SNTLS algorithm on $\tilde{f}_{1}, \ldots, \tilde{f}_{l}$ again until we find the correct $k$.

\section{Algorithms and Experiments}

We have implemented the algorithms in Maple 10 for computing the approximate GCDs of several univariate polynomials with real coefficients by structured low rank approximation of a Bezout matrix. We first present two examples and compare the results with other methods in $[10,22]$.

Example 4.1 [10] Consider the polynomials

$$
\begin{aligned}
& f_{1}=\left(x^{5}-1\right)\left(x^{4}-x+1\right), \\
& f_{2}=\left(x^{5}-0.9999\right)(x+4.0001), \\
& f_{3}=\left(x^{5}-0.9999\right)\left(x^{4}-3.0003 x-2.9999\right), \\
& f_{4}=\left(x^{5}-1.0001\right)\left(x^{4}-3.0001 x-0.9999\right) .
\end{aligned}
$$

The matrix $B_{5}\left(f_{1}, f_{2}, f_{3}, f_{4}\right)$ is of size $27 \times 5$, whereas the generalized Sylvester matrix used in [22] is of size $39 \times 17$. By our algorithms, for $k=5$, after two iterations, we stop the algorithm at $\Delta \mathrm{x}=.28218 \times 10^{-5}$. The deformed polynomials are

$$
\begin{aligned}
\tilde{f}_{1} & =x^{9}+.16753 \times 10^{-5} x^{8}+.26750 \times 10^{-5} x^{7}-x^{6}+x^{5}-.99997 x^{4} \\
& +.19010 \times 10^{-5} x^{3}+.23249 \times 10^{-5} x^{2}+.99998 x-.99998, \\
\tilde{f}_{2} & =.99998 x^{6}+4 x^{5}+.16014 \times 10^{-4} x^{4}+.21290 \times 10^{-4} x^{3}+.17999 \times 10^{-4} x^{2} \\
& -.99992 x-3.9998 \\
\tilde{f}_{3} & =.99996 x^{9}-.10714 \times 10^{-5} x^{8}-.80617 \times 10^{-5} x^{7}-3.0002 x^{6}-2.9998 x^{5} \\
& -.99994 x^{4}-.10723 \times 10^{-5} x^{3}-.80515 \times 10^{-5} x^{2}+3.0001 x+2.9997, \\
\tilde{f}_{4} & =1.0001 x^{9}-.63203 \times 10^{-5} x^{8}-.43391 \times 10^{-5} x^{7}-3.0003 x^{6}-.99998 x^{5} \\
& -x^{4}-.63321 \times 10^{-5} x^{3}-.43314 \times 10^{-5} x^{2}+3.0002 x+0.99992 .
\end{aligned}
$$


The backward error

$$
\mathcal{N}=\sqrt{\left\|\tilde{f}_{1}-f_{1}\right\|_{2}+\left\|\tilde{f}_{2}-f_{2}\right\|_{2}^{2}+\left\|\tilde{f}_{3}-f_{3}\right\|_{2}^{2}+\left\|\tilde{f}_{4}-f_{4}\right\|_{2}^{2}}=.41295 \times 10^{-3} .
$$

The backward error computed by the STLS algorithm in [22] is .41292 $\times 10^{-3}$. However, our algorithm only takes 2.937 seconds while the STLS algorithm takes 213.596 seconds. The backward error given in [10] is larger than $.47610 \times 10^{-3}$. The approximate GCD computed by our algorithm is

$$
\begin{aligned}
c(x)= & x^{5}+.29055 \times 10^{-5} x^{4}+.43923 \times 10^{-5} x^{3}+.37214 \times 10^{-5} x^{2} \\
& +.31134 \times 10^{-5} x-.99995 .
\end{aligned}
$$

Example 4.2 Consider the polynomials [22]

$$
\begin{aligned}
& f_{1}=1000 x^{10}+x^{3}-1, \\
& f_{2}=x^{2}-0.01 .
\end{aligned}
$$

Case 1 If we initialize $\Delta \mathbf{s}=0$ and $\mathbf{x}$ being the unstructured least squares solution of $A(\mathbf{s}) \mathbf{x}=$ $\mathbf{b}(\mathbf{s})$. After 10 iterations, we obtain the deformed polynomials

$$
\begin{aligned}
\tilde{f}_{1} & =1000 x^{10}-0.00011 x^{9}-.00014 x^{8}-.00009 x^{7}+.00008 x^{6}-.00026 x^{5} \\
& +.00049 x^{4}+.99901 x^{3}+.00195 x^{2}-.00386 x-.99238 \\
\tilde{f}_{2} & =.95204 x^{2}+.09462 x-.19666,
\end{aligned}
$$

which have a common divisor $x+0.50690$, and the backward error is

$$
\mathcal{N}=\left\|\tilde{f}_{1}-f_{1}\right\|_{2}^{2}+\left\|\tilde{f}_{2}-f_{2}\right\|_{2}^{2}=0.04617 .
$$

As discussed in [22], this is only one of the local minimum.

Case 2 We initialize $\Delta \mathbf{s}$ by formula (17) and choose $\mathbf{v}$ being the right singular vector corresponding to the smallest singular value of $B_{k}(\mathbf{s})$ and normalized with respect to the largest entry. After 8 iterations, the algorithm returns

$$
\begin{aligned}
\tilde{f}_{1} & =1000 x^{10}+.00012 x^{9}-.00013 x^{8}+.00006 x^{6}+.00023 x^{5}+.00049 x^{4} \\
& +1.0010 x^{3}+.00205 x^{2}+.00415 x-.99156 \\
\tilde{f}_{2} & =.95614 x^{2}-.08876 x-.18962
\end{aligned}
$$

which have a common divisor $x-0.49415$, the backward error is

$$
\mathcal{N}=\left\|\tilde{f}_{1}-f_{1}\right\|_{2}^{2}+\left\|\tilde{f}_{2}-f_{2}\right\|_{2}^{2}=.04216 .
$$

It is the non-monic global minimum similar to the one derived in [22].

In Table 1, we show the performance of our algorithm for computing approximate GCD of univariate polynomials on Pentium 4 at $2.0 \mathrm{Ghz}$ for Digits $=14$ in Maple 10 under 


\begin{tabular}{|r|c|c|c|c|c|c|c|c|}
\hline Ex. & $d_{i}$ & $k$ & $\begin{array}{c}i t . \\
\text { (STLS) }\end{array}$ & $\begin{array}{c}\text { it. } \\
\text { (SNTLS) }\end{array}$ & $\begin{array}{c}\text { error } \\
\text { (STLS) }\end{array}$ & $\begin{array}{c}\text { error } \\
\text { (SNTLS) }\end{array}$ & $\begin{array}{c}\text { time }(s) \\
\text { (STLS) }\end{array}$ & $\begin{array}{c}\text { time }(s) \\
\text { (SNTLS) }\end{array}$ \\
\hline 1 & 2,2 & 1 & 2.18 & 1.90 & $6.96 e-6$ & $6.96 e-6$ & .25 & .12 \\
\hline 2 & 3,3 & 2 & 2.17 & 1.93 & $1.05 e-5$ & $1.07 e-5$ & .31 & .14 \\
\hline 3 & 5,4 & 3 & 2.06 & 1.91 & $1.56 e-5$ & $1.56 e-5$ & .44 & .18 \\
\hline 4 & 5,5 & 3 & 2.27 & 2.00 & $2.04 e-5$ & $2.75 e-5$ & .53 & .20 \\
\hline 5 & 6,6 & 4 & 2.10 & 2.00 & $2.18 e-5$ & $2.18 e-5$ & .58 & .21 \\
\hline 6 & 8,7 & 4 & 2.10 & 1.90 & $1.70 e-5$ & $1.70 e-5$ & .95 & .31 \\
\hline 7 & 10,10 & 5 & 2.60 & 2.10 & $3.43 e-4$ & $3.44 e^{-5}$ & 1.40 & .43 \\
\hline 8 & 14,13 & 7 & 2.60 & 1.90 & $5.73 e-5$ & $6.47 e^{-5}$ & 2.31 & .80 \\
\hline 9 & 28,28 & 14 & 2.00 & 2.00 & $2.60 e-5$ & $2.60 e^{-5}$ & 10.65. & 11.97 \\
\hline 10 & $10,9,8$ & 5 & 4.00 & 3.002 & $7.96 e-5$ & $9.86 e^{-5}$ & 4.17 & 1.99 \\
\hline 11 & $8,7,8,6$ & 4 & 4.40 & 3.20 & $3.24 e^{-5}$ & $3.28 e^{-5}$ & 5.83 & 1.41 \\
\hline
\end{tabular}

Table 1. Algorithm performance on benchmarks (univariate case)

Windows. For every example, we use 50 random cases for each $\left(d_{1}, \ldots, d_{l}\right)$, and report the average over all results. For each example, the prime parts and GCD of polynomials are constructed by choosing polynomials with random integer coefficients in the range $-10 \leq$ $c \leq 10$, and then adding a perturbation. For noise we choose a relative tolerance $10^{-e}$, then randomly choose a polynomial that has the same degree as the product, and coefficients in $\left[-10^{e}, 10^{e}\right]$. Finally, we scale the perturbation so that the relative error is $10^{-e}$. Here $d_{i}$ denotes the degree of the polynomial $f_{i} ; k$ is the degree of the approximate GCD of $f_{1}, \ldots, f_{l}$; it. (STLS) is the number of the iterations needed by method in [23]; whereas it. (SNTLS) denotes the number of iterations by our algorithm; error (STLS) denotes the perturbation $\left\|\tilde{f}_{1}-f_{1}\right\|_{2}^{2}+\cdots+\left\|\tilde{f}_{l}-f_{l}\right\|_{2}^{2}$ computed by algorithm in [23]; whereas error (SNTLS) is the minimal perturbation computed by our algorithm; the last two columns denote the time in seconds costed by two algorithms respectively.

\section{Concluding Remarks}

In this paper we present a new way based on SNTLS to compute the approximate GCD of several univariate polynomials. The overall computational complexity of the algorithm depends on the number of iterations needed for the first order update. If the starting values are good, then the iteration will converge quickly. This can be seen from the above table. Since the matrices involved in the minimization problems are all structured matrix, they have low displacement rank [20]. It would be possible to apply the fast algorithm to solve these minimization problems as in [28]. This would reduce the complexity of our algorithm to be only quadratic with respect to the degrees of the given polynomials.

Our methods can be generalized to several polynomials with arbitrary linear or nonlinear equational constraints imposed on the coefficients of the input and perturbed polynomials. However, at present, our algorithm can't deal with the polynomials with complex coefficients or the global minimal perturbations being complex. Notice that our algorithm also can not 
deal with the case $k=d_{1}$, because in that case the Bezout matrix $B_{k}$ is not defined.

\section{Acknowledgement}

We thank Erich Kaltofen and Zhengfeng Yang for their valuable comments. This work was initiated by the discussions with Robert M. Corless during the Special Semester on Groebner Bases, February 20-24, 2006, organized by RICAM, Austrian Academy of Sciences, and RISC, Johannes Kepler University, Linz, Austria.

\section{References}

[1] S. BARnett, Greatest common divisor of two polynomials, Linear Algebra Appl., 3 (1970), pp. $7-9$.

[2] _ Greatest common divisor of several polynomials, Proc. Camb. Phil. Soc, 70 (1971), pp. 263268.

[3] — A note on the Bezoutian matrix, SIAM J. Appl. Math., 22 (1972), pp. 84-86.

[4] B. Beckermann and G. Labahn, When are two polynomials relatively prime?, J. Symbolic Comput., 26 (1998), pp. 677-689.

[5] D. Bini and L. Gemignani, Fast parallel computation of the polynomial remainder sequence via Bezout and Hankel matrices, SIAM J. Comput., 22 (1993), pp. 63-77.

[6] D. Bini And V. Y. Pan, Polynomial and Matrix Computations, vol. 1 of Fundamental Algorithms, Birkhäuser, 1994.

[7] R. M. Corless, P. M. Gianni, B. M. Trager, and S. M. Watt, The singular value decomposition for polynomial systems, in Proc. 1995 Internat. Symp. Symbolic Algebraic Comput. ISSAC'95, A. H. M. Levelt, ed., New York, N. Y., 1995, ACM Press, pp. 96-103.

[8] R. M. Corless, S. M. Watt, AND L. ZHI, QR factoring to compute the GCD of univariate approximate polynomials, IEEE Transactions on Signal Processing, 52 (2004), pp. 3394-3402.

[9] G. Diaz-Toca And L. Gonzalez-Vega, Barnett's theorems about the greatest common divisor of several univariate polynomials through Bezout-like matrices, J. Symbolic Comput., 34 (2002).

[10] _ Computing greatest common divisors and squarefree decompositions through matrix methods: The parametric and approximate cases, Linear Algebra Appl., 412 (2006).

[11] I. Z. Emiris, A. Galligo, and H. Lombardi, Certified approximate univariate GCDs, J. Pure Applied Algebra., 117 \& 118 (1996), pp. 229-251. Special Issue on Algorithms for Algebra.

[12] F. R. Gantmacher, The Theory of Matrices, vol. 2, Chelsea Publishing Company, 1959.

[13] L. Gemignani, GCD of polynomials and Bezout matrices, in Proc. 1997 (ISSAC'97), W. Küchlin, ed., New York, N. Y., 1997, ACM Press, pp. 271-277.

[14] G. Heining And K. Rost, Algebraic Methods for Toeplitz-like Matrices and Operators, vol. 13 of Operator Theory, Birkhäuser, 1984.

[15] U. Helmke and P. Fuhrmann, Bezoutians, Linear Algebra Appl., 124 (1989), pp. 1039-1097.

[16] M. A. Hitz, On computing nearest singular Hankel matrices, New York, N. Y., 2005, ACM Press, pp. 171-176.

[17] A. Householder, Householder, Bezoutians, elimination and localization, SIAM Review, 12 (1970), pp. 73-78.

[18] V. Hribernig and H. J. Stetter, Detection and validation of clusters of polynomials zeros, J. Symbolic Comput., 24 (1997), pp. 667-681.

[19] J.B.ROSEN, H. PARK, AND J. GLICK, Structure total least norm for nonlinear problems, SIAM J. Matrix Anal. Appl., 20 (1998), pp. 14-30. 
[20] T. Kailath And A. H. SAYed, Displacement structure: theory and applications, SIAM Review, 37 (1995), pp. 297-386.

[21] E. Kaltofen, Z. YAng, And L. Zhi, Structured low rank approximation of a Sylvester matrix. Manuscript, 15 pages, Oct. 2005. Preliminary version in SNC 2005 Proceedings, Dongming Wang and Lihong Zhi eds., pp. 188-201, distributed at the International Workshop on SymbolicNumeric Computation in Xi'an, China, July 19-21, 2005.

[22] — Approximate greatest common divisors of several polynomials with linearly constrained coefficients and singular polynomials, in ISSAC'06 Proc. 2006 Internat. Symp. Symbolic Algebraic Comput., J.-G. Dumas, ed., New York, N. Y., 2006, ACM Press, pp. 169-176.

[23] — Approximate greatest common divisors of several polynomials with linearly constrained coefficients and singular polynomials. Manuscript, 20 pages, Dec 2006.

[24] N. Karmarkar And Lakshman Y. N., Approximate polynomial greatest common divisors and nearest singular polynomials, in Proc. 1996 Internat. Symp. Symbolic Algebraic Comput. (ISSAC'96), Lakshman Y. N., ed., New York, N. Y., 1996, ACM Press, pp. 35-42.

[25] P. Lemmerling, Structured total least squares: analysis, algorithms and applications, dissertation, Katholieke Universiteit Leuven, Belgium, 1999.

[26] P. Lemmerling, N. Mastronardi, and S. V. Huffel, Fast algorithm for solving the Hankel/Teoplitz strucured total least squares problem, Numerical Algorithms, 23 (2000), pp. 371-392.

[27] H. LeV-Ari, Y. Bistritz, And T. Kailath, Generalized Bezoutians and families of efficient zero-location procedures, IEEE Transactions on Circuits and Systems, 38 (1991), pp. 170-186.

[28] B. LI, Z. YANG, AND L. ZHI, Fast low rank approximation of a Sylvester matrix by structured total least norm, J. JSSAC (Japan Society for Symbolic and Algebraic Computation), 11 (2005), pp. $165-174$.

[29] M. T. Noda And T. SAsaki, Approximate GCD and its application to ill-conditioned algebraic equations, J. Comput. Appl. Math., 38 (1991), pp. 335-351.

[30] V. Y. PAN, Numerical computation of a polynomial GCD and extensions, Information and computation, 167 (2001), pp. 71-85.

[31] H. PARK, L. ZHANG, AND J. B. Rosen, Low rank approximation of a Hankel matrix by structured total least norm, BIT, 39 (1999), pp. 757-779.

[32] J. B. Rosen, H. PARK, And J. Glick, Total least norm formulation and solution for structured problems, SIAM J. Matrix Anal. Appl., 17 (1996), pp. 110-128.

[33] D. Rupprecht, An algorithm for computing certified approximate gcd of $n$ univariate polynomials, J. Pure Appl. Algebra, 139 (1999), pp. 255-284.

[34] M. SASAKI AND T. SASAKI, Polynomial remaider sequence and approximate GCD, ACM SIGSAM Bulletin, 31 (2001), pp. 4-10.

[35] A. Schönhage, Quasi-gcd computations, Journal of Complexity, 1 (1985), pp. 118-137.

[36] D. Sun And L. ZHI, Structured low rank approximation of a Bezout matrix, Dec 2006. MMRC Preprint. Extended abstract appeared at the Proc. of International Conference on Mathematics Aspects of Computer and information sciences.

[37] Z. ZENG, The approximate GCD of inexact polynomials. part I: a univariate algorithm. Manuscript, 2004.

[38] Z. ZENG, Computing multiple roots of inexact polynomials, Math. Comput., 74 (2005), pp. 869903.

[39] L. ZHI, Displacement structure in computing approximate GCD of univariate polynomials, in Proc. Sixth Asian Symposium on Computer Mathematics (ASCM 2003), Z. Li and W. Sit, eds., vol. 10 of Lecture Notes Series on Computing, Singapore, 2003, World Scientific, pp. 288-298. 
[40] L. Zhi And M. T. NodA, Approximate GCD of multivariate polynomials, in Proc. Fourth Asian Symposium on Computer Mathematics (ASCM 2000), X. Gao and D. Wang, eds., vol. 8 of Lecture Notes Series on Computing, Singapore, 2000, World Scientific, pp. 9-18. 